important by patients, not by experts, it entails the risk that experts will not submit research proposals on these topics. This shows once more, the importance to consider patient participation in research agenda setting as a mutual learning process for patients, experts and policymakers.

Janneke Elberse*, Dorothee Laan", Tjard de Cock Buning*, Truus Teunissen ${ }^{\#}$, Jacqueline Broerse* and Willem de Boer ${ }^{\#}$ *Athena Institute, VU University, Amsterdam, and ${ }^{*}$ Netherlands Asthma Foundation, Amersfoort, The Netherlands.

Correspondence: J. Elberse, Athena Institute, VU University Amsterdam, De Boelelaan 1085, 1081 HV Amsterdam, the Netherlands. E-mail: j.e.elberse@vu.nl

Statement of Interest: None declared.

Acknowledgements: The authors are grateful for the willingness of the patients and experts who were involved in the process. Their contribution has been highly valued. Also the collaboration with the Asthma Foundation lung patients association, Dutch Sarcoidosis interest group, Dutch PHA Foundation, Dutch Cystic Fibrosis Foundation and the Dutch Pulmonary Fibrosis organisation was of great value to us.

\section{REFERENCES}

1 Caron-Flinterman JF, Broerse JEW, Bunders JFG. Patient partnership in decision-making on biomedical research: changing the network. Sci Technol Hum Val 2007; 32: 339-368.

2 Entwistle VA, Renfrew MJ, Yearley S, et al. Lay perspectives: advantages for health research. BMJ 1998; 316: 463-466.

3 Tallon D, Chard J, Dieppe P. Relation between agendas of the research community and the research consumer. Lancet 2000; 355: 2037-2040.

4 Telford R, Beverley CA, Cooper CL, et al. Consumer involvement in health research: fact or fiction? Br J Clinical Gov 2002; 7: 92-103.

5 Lloyd K, White J. Democratizing clinical research. Nature 2011; 474: 277-278.

6 Stewart RJ, Caird J, Oliver K, et al. Patients' and clinicians' research priorities. Health Expect 2011; 14: 439-448.

7 Caron-Flinterman JF, Broerse JE, Teerling J, et al. Patients' priorities concerning health research: the case of asthma and COPD research in the Netherlands. Health Expect 2005; 8: 253-263.

8 Caron-Flinterman JF, Broerse JEW, Teerling J, et al. Stakeholder participation in health research agenda setting: the case of asthma and COPD research in the Netherlands. Sci Public Policy 2006; 33: 291-304.

9 Abma TA, Broerse JEW. Patient participation as dialogue: setting research agendas. Health Expect 2010; 13: 160-173.

10 Cornwall A, Jewkes R. What is participatory research? Soc Sci Med 1995; 41: 1667-1676.

\title{
Viral infections trigger exacerbations of cystic fibrosis in adults and children
}

\section{To the Editors:}

Cystic fibrosis (CF) is the most common autosomal recessive condition causing disease in western societies, and despite important advances in understanding the disease, patients with CF develop progressive lung disease with recurrent endobronchial infection, eventually becoming chronically colonised with resistant organisms such as Pseudomonas aeruginosa. The clinical course is punctuated by periods of acute worsening of CF lung disease that increase with age and declining lung function, while the frequency of exacerbations is also an independent predictor for decline in lung function and mortality [1]. How and why exacerbations of CF occur is poorly understood. Recent data suggest that exacerbations are not associated with an acquisition of new strains of bacteria, but rather clonal expansion of existing strains [2]. The factors that lead to this imbalance between chronic bacterial infection and host immune response, which then results in CF exacerbations, are unclear. Viral infection may be an important factor that triggers these events. In children with $\mathrm{CF}$, viral respiratory tract infections are associated with exacerbations [3, 4]. In CF a respiratory virus infection superimposed upon chronic bacterial infection potentially could enhance inflammation and shift the balance to favour infection with chronic bacterial pathogens.
The aims of this study were to assess the prevalence and aetiology of viral respiratory tract infection in an adult population with $\mathrm{CF}$, compare this to a group of children with CF and assess the impact of this on acute exacerbations of lung disease.

We recruited 17 adults (age greater than $17 \mathrm{yrs}$ ) and nine children (6-17 yrs) from the CF clinic at John Hunter Hospital, New Lambton, Australia with CF diagnosed by a positive sweat test and CF genotyping. Participants were assessed at baseline and reviewed every 3 months for 1 yr, from 2007 to 2009. Participants were advised to contact investigators as soon as they developed symptoms of a cold or a worsening of their chest disease and reviewed in $48 \mathrm{~h}$. Acute exacerbations were defined when any four of the 12 items of the Fuchs' criteria were present [5]. At each visit, spontaneous sputum was sent for quantitative microbiology [6] and two throat swabs were performed using a semi-nested PCR for influenza A and B, respiratory syncytial virus (RSV), rhinovirus (RV), coronavirus, human metapneumovirus, parainfluenza 1, 2 and 3, and adenovirus [7] and RV were sequenced [8]. When data were normally distributed, differences were analysed using a non-paired t-test and, when not normally distributed, a non-parametric equivalent. Multivariate linear regression analysis was performed to determine risk for acute exacerbation, risk for exacerbation 
requiring hospitalisation and change in forced expiratory volume in $1 \mathrm{~s}$ (FEV1) over the time of the study.

In the 17 adults, 11 out of 17 were male, with mean age 27.5 yrs. They had moderate airflow obstruction, with mean (range) FEV1 $62.5 \%$ predicted (38-98\% pred). All had chronic bacterial infection on sputum culture, 13 out of 17 with $P$. aeruginosa, one out of 17 Burkholderia cenocepacia, and five out of 17 with Staphylococcus aureus (methicillin sensitive). Regular azithromycin was used in 13 out of 17 and nebulised tobramycin in four out of 17; 11 out of 17 had received influenza vaccination that year. All were pancreatic insufficient, mean \pm SD body mass index (BMI) 22.3 \pm 2.9 ; seven out of 17 had diabetes.

The nine children had a mean \pm SD age of $10.2 \pm 3.9$ yrs; they had normal or only mild airflow obstruction, with mean (range) FEV1 $89.0 \%$ pred (74-105\% pred). Only three out of nine had chronic colonisation with $P$. aeruginosa, and two out of nine with $S$. aureus (methicillin sensitive). Regular azithromycin was used in two out of nine, regular flucloxacillin in two out of nine and nebulised tobramycin one out of nine; seven out of nine had been vaccinated for influenza. There were 11 out of 17 (64\%) adults who received influenza vaccination that year. All were pancreatic insufficient, mean \pm SD BMI 19.6 \pm 2.4 ; none had diabetes.

In adults, 14 out of 17 participants reported an increase in chest symptoms or colds resulting in 38 episodes, only three participants had no episodes. Of the 38 reported episodes, $25(66 \%)$ met the definition of an exacerbation; 15 did not meet the criteria and did not require a change in treatment, though one re-presented and was treated. Of the adult exacerbations, seven out of $25(28 \%)$ required hospitalisation and were treated with intravenous antibiotics; two out of 38 had i.v. antibiotics commenced as an outpatient, 15 out of 25 were treated with oral and nebulised antibiotics.

In adults a virus was isolated by PCR in 20 out of $38(52 \%)$ presentations and was associated with 17 out of $25(68 \%)$ exacerbations. The predominant viruses detected were RV (12 episodes, nine exacerbations), the most prevalent belonging to the RV-A clade (exacerbations; RV-A seven, RV-B one, RV-C one). The other viruses detected were all associated with exacerbations; influenza three, RSV one and coronavirus with four.

There were seven children who reported an increase in chest symptoms or colds resulting in 18 episodes of increased symptoms. Of the 18 episodes, five were exacerbations. The exacerbations were treated as follows: two out of five required treatment with i.v. antibiotics in hospital and three out of five were treated with oral antibiotics.

In the children, virus detection was associated with 13 out of 18 (72\%) episodes with increased respiratory symptoms or colds (more frequently seen than in the adults; $\mathrm{p}=0.02$ ) and all five exacerbations. RVs again were predominant (11 episodes; RV-A nine, RV-B two), RV-A was associated with four exacerbations, while RSV was associated with one. There were no viruses detected in samples that were taken at baseline or when participants were stable.

A regression analysis was performed to determine which variables were associated with an increased risk of exacerbation. This was assessed in the presence of diabetes, lung function (FEV1\% pred), age, sex, BMI, the presence of bacterial infection on sputum culture at baseline, and virus detected at presentation. We determined that lower BMI $(p=0.04)$, diabetes $(p=0.009)$, the presence of $P$. aeruginosa at baseline $(p=0.004)$, lower FEV1 $(p=0.02)$ and the presence of virus $(p=0.04)$ all predicted an exacerbation occurring, accounting for $62 \%$ of the variation in exacerbation frequency (adjusted $\mathrm{R}^{2}=0.62 ; \mathrm{p}=0.017$ ).

In those who did exacerbate, virus infection was not associated with a greater fall in lung function (fig. 1a) at presentation. Virus infection was associated with an increase in $P$. aeruginosa $\mathrm{CFU} \cdot \mathrm{mL}^{-1}$ (fig. 1b). There were no subjects who acquired a new bacterial pathogen in their sputum during the course of the study.
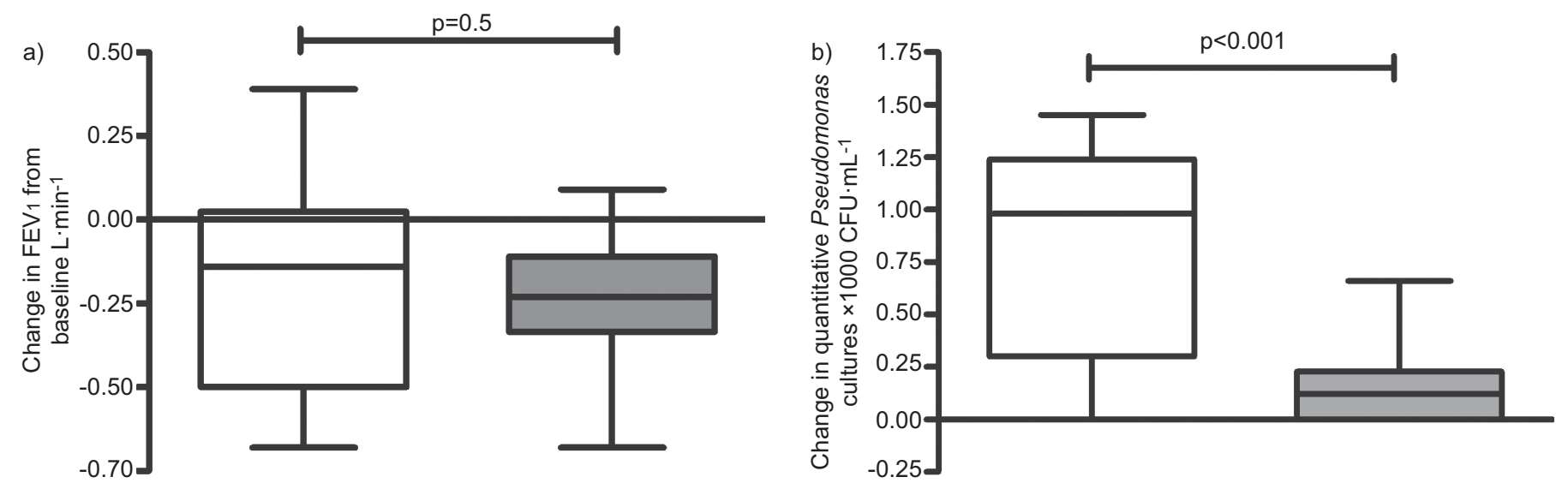

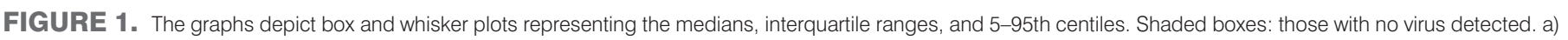

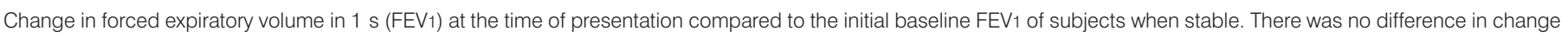

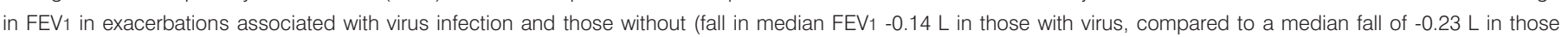

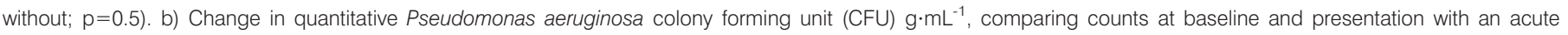

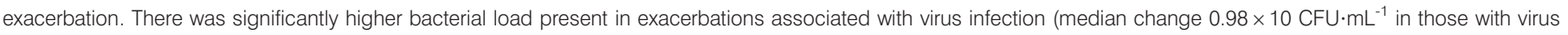
infection, versus $0.12 \times 10 \mathrm{CFU} \cdot \mathrm{mL}^{-1}$ in those without; $\mathrm{p}=0.01$ ). The analysis was limited to adult subjects only. 
Viral respiratory tract infections were associated with $65 \%$ of CF exacerbations in children and adults, with RV A the most frequently isolated. Virus infection was also associated with an acute increase in $\mathrm{CFU} \cdot \mathrm{mL}^{-1}$ of $P$. aeruginosa in those with chronic infection. This is intriguing as virus infection may also influence the status quo in the CF airway between chronic infection with $P$. aeruginosa and the host. In vitro RV infection has been shown to liberate Pseudomonas from biofilms, planktonic bacteria that are then more pro-inflammatory [9]. This observation would suggest this proposed mechanism is important in the pathogenesis of acute CF exacerbations.

In conclusion, virus infections are important precipitants of $\mathrm{CF}$ exacerbations, though the risk that they will trigger an exacerbation is determined in the context of the severity of preexisting pulmonary and comorbid disease. Given the importance of exacerbations in worsening $\mathrm{CF}$, further work needs to be done to examine the role of virus infection and how this influences airway inflammation and chronic infection. Consideration should also be given to clinical interventions that may reduce the effect of viruses in $\mathrm{CF}$, this includes the role and effectiveness of influenza vaccination, but should also consider the effectiveness of infection control practices for families and individuals to reduce virus acquisition.

Peter A.B. Wark*, , Melinda Tooze ${ }^{\star}$, Linda Cheese ${ }^{+}$, Bruce Whitehead $^{+}$, Peter G. Gibson ${ }^{\#}$, Katrina F. Wark ${ }^{\#}$ and Vanessa M. McDonald ${ }^{\#, \S, f}$

*University of Newcastle, Centre for Asthma and Respiratory Diseases, "Hunter Medical Research Institute - Respiratory and Sleep Medicine, ${ }^{\S}$ School of Nursing and Midwifery, The University of Newcastle, ${ }^{f}$ School of Medicine and Public Health, The University of Newcastle, Newcastle, "Dept of Respiratory and Sleep Medicine, John Hunter Hospital, and ${ }^{+}$Dept of Respiratory Medicine, John Hunter Children's Hospital, New Lambton, Australia.
Correspondence: P.A.B. Wark, University of Newcastle Centre for Asthma and Respiratory Disease, Dept of Respiratory and Sleep Medicine, John Hunter Hospital, Lookout Rd, New Lambton, New South Wales 2305, Australia. E-mail: peter.wark@hnehealth.nsw.gov.au

Statement of Interest: A statement of interest for P.A.B. Wark can be found at www.erj.ersjournals.com/site/misc/statements.xhtml

\section{REFERENCES}

1 de Boer K, Vandemheen KL, Tullis E, et al. Exacerbation frequency and clinical outcomes in adult patients with cystic fibrosis. Thorax 2011; 66: 680-685.

2 Aaron SD, Ramotar K, Ferris W, et al. Adult cystic fibrosis exacerbations and new strains of Pseudomonas aeruginosa. Am J Respir Crit Care Med 2004; 169: 811-815.

3 Armstrong D, Grimwood K, Carlin JB, et al. Severe viral respiratory infections in infants with cystic fibrosis. Pediatr Pulmonol 1998; 26: 371-379.

4 Smyth A, Lewis S, Bertenshaw C, et al. Case-control study of acute renal failure in patients with cystic fibrosis in the UK. Thorax 2008; 63: 532-535.

5 Fuchs HJ, Borowitz DS, Christiansen DH, et al. Effect of aerosolized recombinant human DNase on exacerbations of respiratory symptoms and on pulmonary function in patients with cystic fibrosis. N Engl J Med 1994; 331: 637-642.

6 Burns JL, Emerson J, Stapp JR, et al. Microbiology of sputum from patients at cystic fibrosis centers in the United States. Clin Infect Dis 1998; 27: 158-163.

7 Wark PAB, Johnston SL, Moric I, et al. Neutrophil degranulation and cell lysis is associated with clinical severity in virus-induced asthma. Eur Respir J 2002; 19: 68-75.

8 Palmenberg AC, Spiro D, Kuzmickas R, et al. Sequencing and analyses of all known human rhinovirus genomes reveal structure and evolution. Science 2009; 324: 55-59.

9 Chattoraj SS, Ganesan S, Jones AM, et al. Rhinovirus infection liberates planktonic bacteria from biofilm and increases chemokine responses in cystic fibrosis airway epithelial cells. Thorax 2011; 66: 333-339.

DOI: $10.1183 / 09031936.00202311$ 